\title{
Ultra-Heavy Cosmic Ray Analysis with CALET on the International Space Station: Established and Developing Procedures
}

\section{A. W. Ficklin, ${ }^{1, *}$ N. Cannady, ${ }^{2,3,4}$ B. F. Rauch ${ }^{5}$ and W. Zober ${ }^{5}$ on behalf of the CALET Collaboration Collaboration \\ (a complete list of authors can be found at the end of the proceedings)}

${ }^{1}$ Department of Physics and Astronomy, Louisiana State University, 202 Nicholson Hall, Baton Rouge, Louisiana 70803, USA

${ }^{2}$ Center for Space Sciences and Technology, University of Maryland, Baltimore County, 1000 Hilltop Circle, Baltimore, Maryland 21250, USA

${ }^{3}$ Astroparticle Physics Laboratory, NASA/GSFC, Greenbelt, Maryland 20771, USA

${ }^{4}$ Center for Research and Exploration in Space Sciences and Technology, NASA/GSFC, Greenbelt, Maryland 20771, USA

${ }^{5}$ Department of Physics and McDonnell Center for the Space Sciences, Washington University, One Brookings Drive, St. Louis, Missouri 63130-4899, USA

E-mail: afick13@lsu.edu

The CALorimetric Electron Telescope (CALET) has collected over 60 months of uninterrupted data on the flux and spectrum of the Ultra-Heavy (UH) cosmic rays from $\mathrm{Z}=30$ to 40 . Using the latest data provided from CALET's UH trigger, we present a newly developed UH analysis complementary to the ongoing analysis presented at this conference by Zober et al. This work introduces a new Ultra-Heavy Analysis (UHA) dataset produced from CALET production data allowing for more streamlined analysis. We detail temporal and spatial correction algorithms using both the ${ }_{26} \mathrm{Fe}$ and ${ }_{14} \mathrm{Si}$ peaks to improve charge resolution in the $\mathrm{Z}>=30$ region. Additionally, this work presents a new method for removing the contributions from non-relativistic/lower-Z nuclei using trajectory tracing to compute effective cutoff rigidities in place of the previously used vertical Stoermer approximation. We show that replacing the approximated cutoffs with numerically calculated effective cutoff rigidities, calculated using the IGRF13 and T05 (Tsyganenko 05) geomagnetic field models, leads to fewer events being removed from the dataset while maintaining improved charge resolution for $Z>26$. Furthermore, we introduce Tarle function peak fitting to perform charge corrections needed as a result of any quenching effects. We show the most recent CALET UH results incorporating these improvements in the analysis

$37^{\text {th }}$ International Cosmic Ray Conference (ICRC 2021)

July 12 th - 23rd, 2021

Online - Berlin, Germany

\footnotetext{
${ }^{*}$ Presenter
} 


\section{Introduction}

The CALorimetric Electron Telescope (CALET) has collected over 60 months of uninterrupted data on the flux and spectrum of the Ultra-Heavy (UH) cosmic rays from $\mathrm{Z}=30$ to 40 . Using the latest data provided from CALET's UH trigger, this work presents a newly developed UH analysis complementary to the ongoing analysis by fellow CALET collaborators. We detail temporal and spatial correction algorithms using both the ${ }_{26} \mathrm{Fe}$ and ${ }_{14} \mathrm{Si}$ peaks to improve charge resolution in the $\mathrm{Z}>=30$ region. Additionally, this work presents a new method for removing contamination from non-relativistic/lower-Z nuclei using trajectory tracing to compute effective cutoff rigidities in place of the previously used vertical Stoermer approximation. We show that replacing the approximated cutoffs with numerically calculated effective cutoff rigidities, calculated using the IGRF13 and T05 (Tsyganenko 05) geomagnetic field models, leads to fewer events being removed from the dataset while maintaining improved charge resolution for $Z>26$. Furthermore, we introduce Tarle function peak fitting to perform charge corrections needed as a result of any quenching effects. We show the most recent CALET UH results incorporating these improvements in the analysis.

\section{The CALET Instrument}

CALET was launched in August 2015 to the International Space Station (ISS) for the primary purpose of directly measuring the cosmic-ray total electron spectrum up to energies of tens of TeV. The CALET calorimeter (CAL) [2], shown in Figure 1, has a depth of 30 radiation lengths $\left(X_{0}\right)$ at normal incidence and includes three separate sub-detectors. The Charge Detector (CHD) consists of two layers of 14 plastic scintillating paddles and can measure the charge of nuclei up to $\mathrm{Z}=40$. The Imaging Calorimeter (IMC) contains 16 layers of 448 scintillating fibers each which provide accurate tracking for most events. Most of the shower energy is deposited in the Total Absorption Calorimeter (TASC), with 12 layers of 16 PWO logs that can contain electromagnetic showers up to TeV energies.

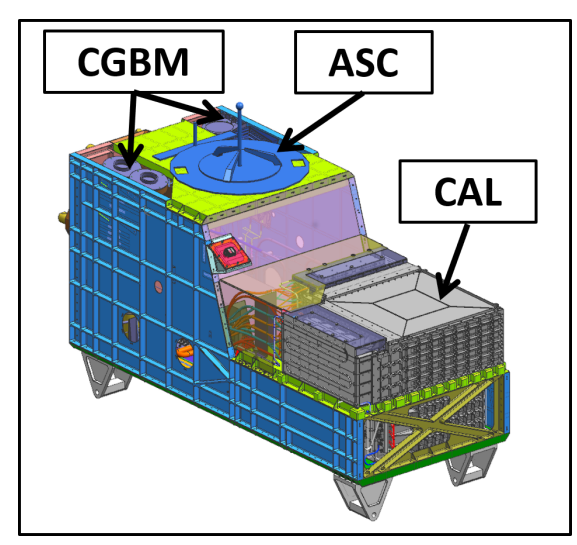

Figure 1: CALET structure indicating the CALET Gamma-ray Burst Monitor (CGBM), Advanced Stellar Compass (ASC), and calorimeter (CAL).

For ultra-heavy cosmic ray observations CALET uses a dedicated trigger mode that is active throughout the ISS orbit [1]. Standard trigger modes require events pass through the TASC, limiting the field of view, but the UH trigger removes this restriction and only requires that events pass through the CHD and top half of the IMC [1]. This raises the geometric factor from $\sim 1200 \mathrm{~cm}^{2} s r$ to $\sim 4000 \mathrm{~cm}^{2} s r$, and prevents UH event candidates similar to the one shown in Figure 2 from being removed from the dataset. 


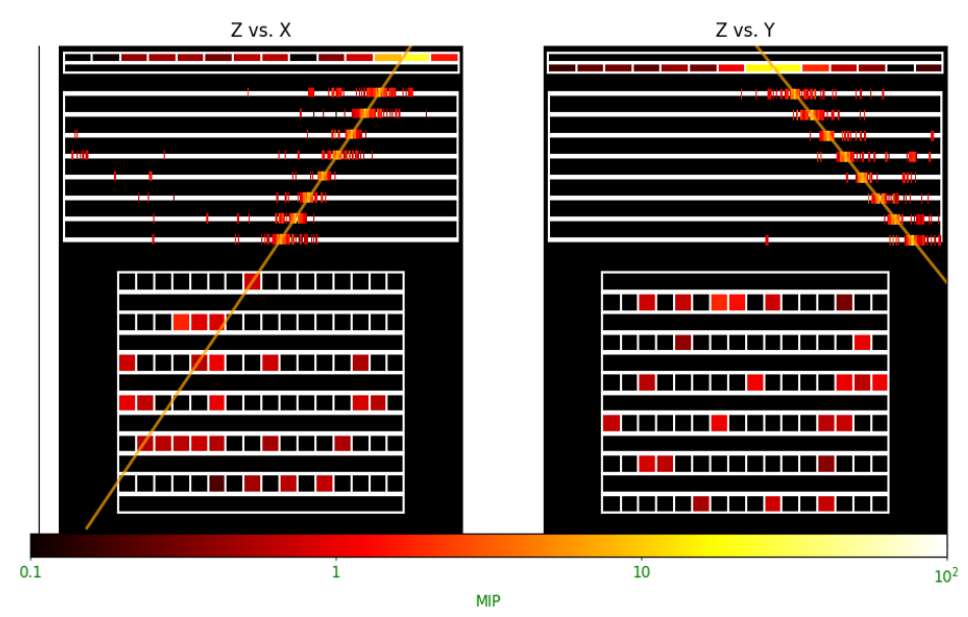

Figure 2: Ultra-heavy event candidate shown in the CALET event viewer. The calculated UH track is shown by the orange line.

\section{Data Analysis}

\subsection{Ultra-Heavy Analysis Dataset}

To aid the UH data analysis a reduced high level analysis UH dataset was produced using 5 years of the CALET Level 2 Pass 4.1 dataset from October 2015 to September 2020. Included for each of the $1.8 \times 10^{8}$ events is the associated summary information, energy-deposit sums for each layer of the instrument, orbital parameters of the ISS at the time of observation, UH track reconstruction parameters, and individual energy deposits for detector elements on or near the calculated track. The estimated charge of each event is calculated using the event's $d E / d x$ and the Bethe equation, which reduces to Equation 1 assuming the particle is minimum ionizing.

$$
\frac{d E}{d x} \propto z^{2}
$$

A selection is then made to the initial set of events requiring their reconstructed track's incidence angle be less than 60 degrees [3]. These high incidence angle events can have their charges over estimated resulting in a reduction of charge resolution in the UH region. A charge consistency cut [3] is applied to the remaining events removing events where the estimated charges in the $\mathrm{x}$ and $\mathrm{y}$ layers of the CHD (CHDX amd CHDY) differ by greater than 1.5\%. The final dataset used for this analysis includes $5 \times 10^{7}$ events.

\subsection{Charge Corrections}

\subsubsection{Time/Spatial Dependence}

When calculating charge using the method described above, long-term changes in temperature and PMT gain cause time dependent fluctuations that negatively impact charge resolution. To address these variations, an example of which is shown in the left side of Figure 3, events are placed in 1-day bins and fits performed to find the location of the ${ }_{26} \mathrm{Fe}$ and ${ }_{14} \mathrm{Si}$ peaks for each paddle of both CHDX and CHDY. These peaks are then used to calculate linear correction parameters for 
each day of data. When the corrections are applied, each bin's charge spectrum is linearly shifted so that the new ${ }_{26} \mathrm{Fe}$ and ${ }_{14} \mathrm{Si}$ peak locations are equal to their mean values for the full 5-year dataset.

Further variation in charge, shown in the right of Figure 3, arises from light propagation effects and non-uniformity in the CHD that both contribute to a spatial dependence in the light yield of the detector. To account for this dependence, the time-corrected data is divided into $3.2 \mathrm{~cm} \times 3.2 \mathrm{~cm}$ bins in both the CHDX and CHDY. For each bin, the fitted ${ }_{26} \mathrm{Fe}$ and ${ }_{14} \mathrm{Si}$ peak locations are again used to calculate the linear correction needed to shift each peak to its mean value for the entire CHDX and CHDY respectively.
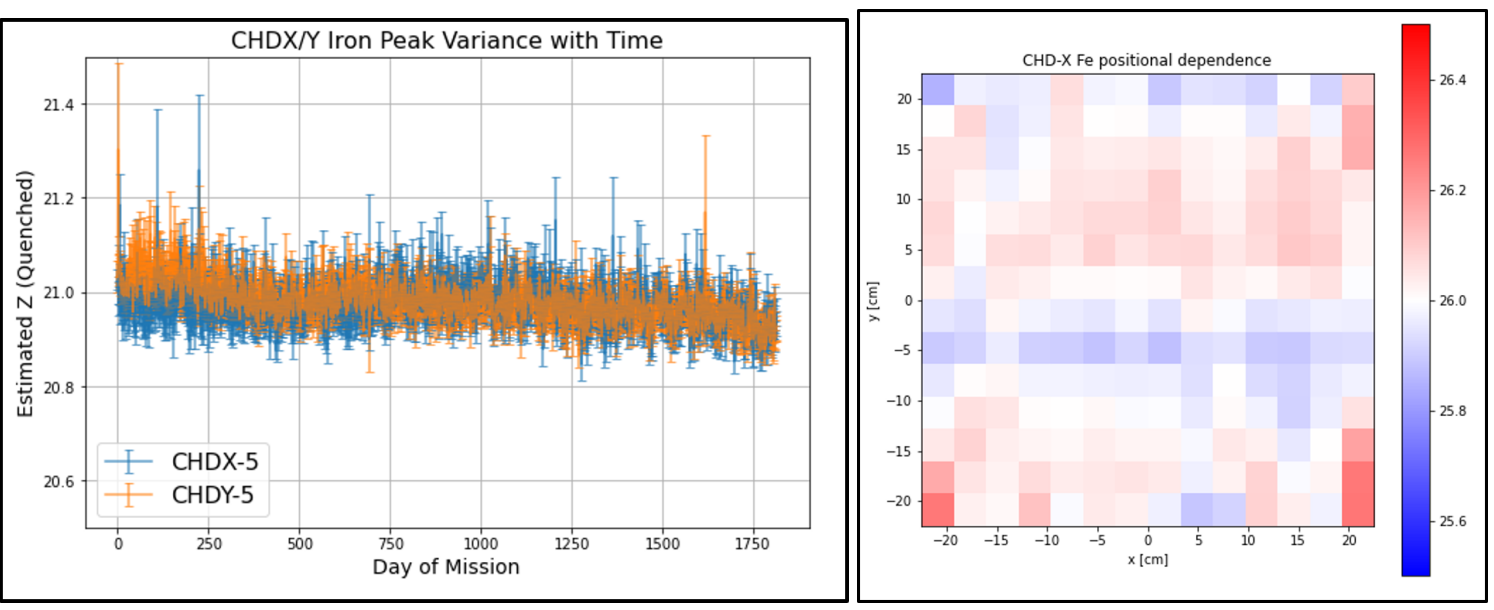

Figure 3: left: Location of the ${ }_{26} \mathrm{Fe}$ peak for paddle 5 of both CHDX and CHDY as a function of time. right: Location of the ${ }_{26} \mathrm{Fe}$ peak for each position bin of the CHDX.

\subsubsection{Quenching Correction}

In organic scintillators, it is found that large deposits of energy that are spatially concentrated will exhibit non-linear saturation of the scintillator response, or quenching. This results in underestimated charge assignments that worsens as $Z$ increases. Using a method derived by Tarle et al., it is possible to recover the true $d E / d x$ from the actual luminosity, $d L / d x$, via

$$
\frac{d L}{d x}=\mathrm{K} \frac{\left(1-F_{s}\right) d E / d x}{1+B\left(1-F_{S}\right) d E / d x}+F_{s} \frac{d E}{d x},
$$

where $F_{s}$ describes the fraction of electrons produced in the ionization escape between the saturated core and unsaturated halo regions of the signal, and $\mathrm{B}$ and $\mathrm{K}$ are constants. Combining Equations 1 and 2 , we get

$$
\frac{d L}{d x}=T\left(Z^{2}\right)=\frac{B_{1} Z^{2}}{1+B_{2} Z^{2}}+B_{3} Z^{2} .
$$

Inverting this result then lets us solve for the un-quenched $d E / d x$ directly from our measured luminosity, $d L / d x$, using

$$
Z^{2}=\frac{1}{2 B_{2} B_{3}}\left[\left(\frac{d L}{d x} B_{2}-B_{3}-B_{1}\right)+\sqrt{\left(\frac{d L}{d x} B_{2}-B_{3}-B_{1}\right)^{2}+4 \frac{d L}{d x} B_{2} B_{3}}\right],
$$


where $B_{1}, B_{2}$, and $B_{3}$ are fit constants. To calculate the necessary Tarle function parameters, we performed a multi-peak fit for all nuclei from ${ }_{6}$ to ${ }_{28} \mathrm{Ni}$ and fit these directly with the Tarle function. The result of applying this quenching correction is shown in Figure 4.

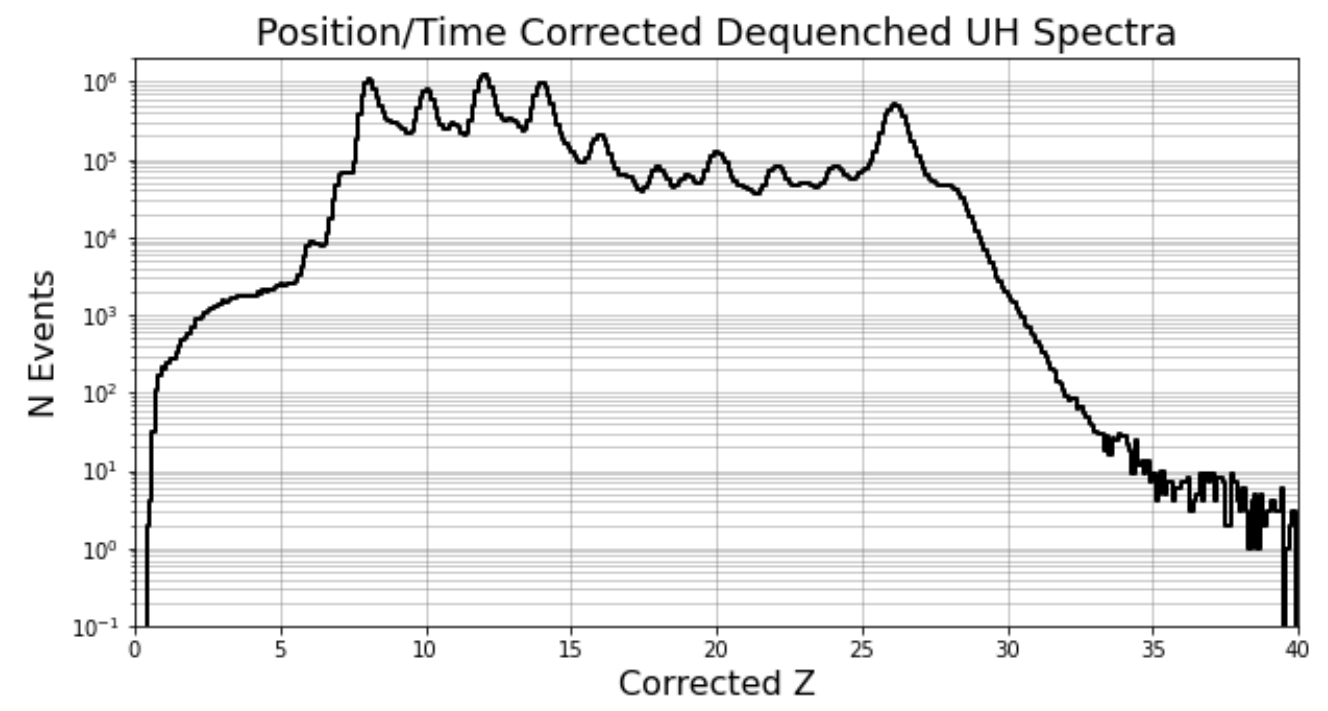

Figure 4: Quenching corrected CHDX charge spectra for time and position corrected UH events.

\subsection{Charge Resolution Improvements}

\subsubsection{Stoermer Cutoff Rigidity}

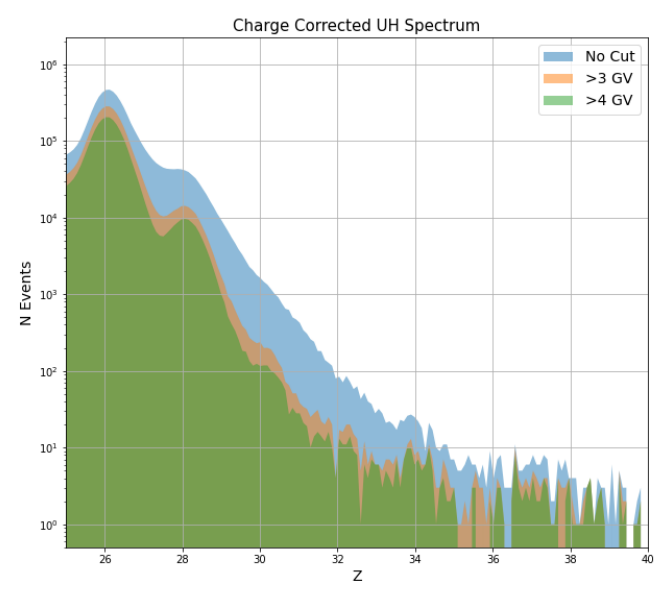

Figure 5: Charge corrected UH spectrum with no cut, a cut at $R>3 \mathrm{GV}$, and a cut at $R>4 \mathrm{GV}$. Where $\mathrm{R}$ is the vertical cutoff rigidity.
To achieve adequate charge resolution in the $\mathrm{UH}(\mathrm{Z}>=30)$ region, a method was required for removing the primary source of contamination, non-relativistic and lower $\mathrm{Z}$ nuclei that are below minimum ionizing and consequently have significantly increased $\frac{d E}{d x}$. Given CALET's orbit is deep within the Earth's geomagnetic field and experiences a wide range of cutoff rigidities, implementing a cut using geomagnetic cutoff rigidity was the chosen method. Non-relativistic nuclei below minimum ionization have access to the instrument through a smaller, lower range of rigidities than higher energy nuclei, so removing times where the cutoff rigidity is in this range limits their inclusion in the dataset.

Initially, Stoermer's vertical cutoff rigidity approximation [4], Equation 5, was used for determining cutoffs where $\Psi$ is magnetic latitude. 


$$
R_{c}=14.9 * \cos ^{4}(\Psi)
$$

Figure 5 shows the results of implementing a cut using this approximated cutoff, showing cut thresholds of $3 \mathrm{GV}$ and $4 \mathrm{GV}$. Although there is visible improvement in the charge resolution, the loss of statistics in the UH region limits quickly becomes prohibitive to the analysis.

To refine the calculation of the cutoff rigidties, the vertical Stoermer approximation was replaced with its trajectory dependent equivalent [4]. Instead of assuming normal incidence, Equation 6 now takes into account the incident particle's zenith, $\phi$, and azimuth, $\theta$, angles, as well as its distance from the magnetic dipole, $r$, when deriving the cutoff. A comparison between the computed cutoff rigidties is shown in Figure 6, and shows significant divergence between the vertical and trajectory dependent values.

$$
R_{c}=\frac{60}{r^{2}} \times\left(\frac{1-\sqrt{1-\cos (\Psi) \cos ^{3}(\theta)}}{\cos (\Psi) \cos (\theta)}\right)^{2}
$$

This divergence though appears primarily at times of high cutoff rigidity, and does not affect events observed over much of the orbit.

\subsubsection{Numerical Method for Calculating Cutoff}

To improve on the Stoermer approximation, a trajectory tracing method developed by Smart and Shea [5] was implemented to numerically solve for effective cutoff rigidities on an event by event basis. For each trajectory, the method uses an adaptive step size Runge-Kutta method to integrate the equations of motion for a charged particle in a magnetic field, stepping through the trajectory until it is deemed successful or forbidden. This is repeated for a range of rigidity values for each event, resulting in a spectrum of allowed and forbidden rigidities. An example of this, shown in Figure 7, shows all allowed rigidities in white and forbidden rigidities in blue. Notable points are the upper

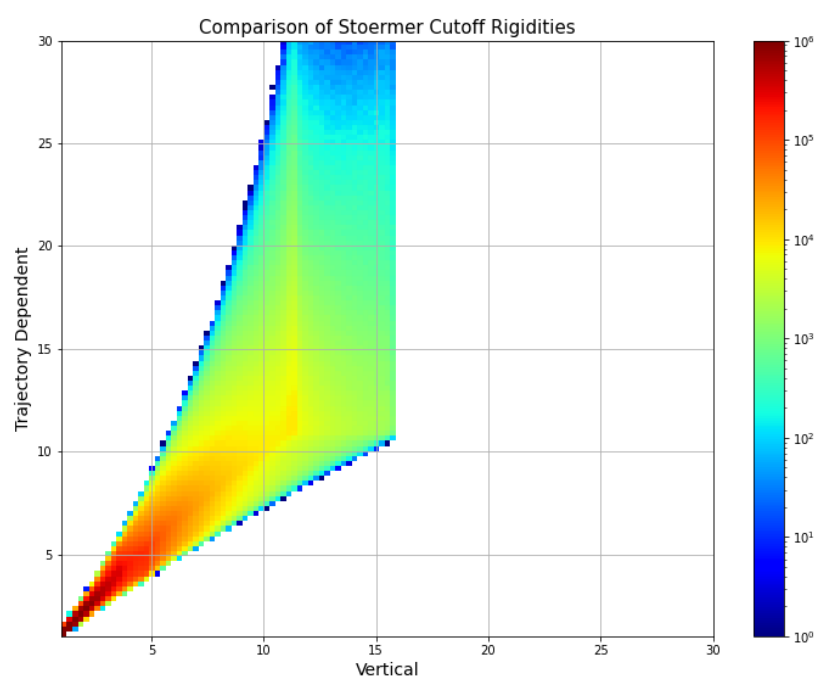
cutoff rigidity where the first forbid-

Figure 6: Comparison between trajectory dependent and vertical Stoermer cutoff rigidities. den trajectory occurs, and the lower rigidity where the last allowed rigidity occurs. The effective cutoff rigidity is calculated using

$$
R_{E}=R_{L}+\left(R_{U}-R_{L}\right) \times N_{\text {Forbidden }} / N_{\text {Total }},
$$




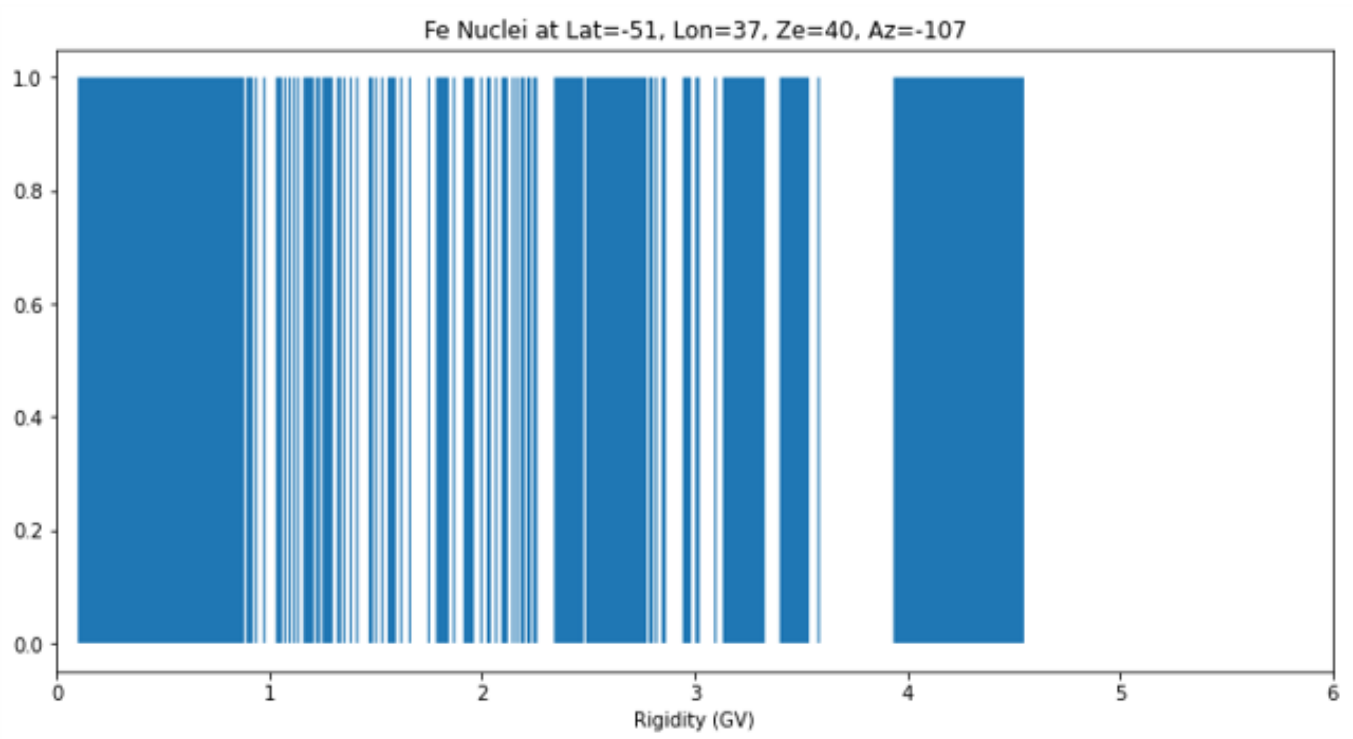

Figure 7: Spectrum of allowed and forbidden trajectories for an example ${ }_{26} \mathrm{Fe}$ event trajectory, with allowed trajectories being white space, and forbidden trajectories being blue space.

where $R_{L}$ is the lower cutoff, $R_{U}$ is the upper cutoff, $N_{\text {Forbidden }}$ is the number of forbidden trajectories after $R_{U}$, and $N_{\text {Total }}$ is the total number of trajectories between $R_{U}$ and $R_{L}$. This effective rigidity is the value used for the calculated cutoff rigidity, as it takes into account the effect of the penumbra (allowed trajectories interspersed amongst forbidden trajectories) and is a more appropriate value when compared to the upper or lower cutoff. The effective cutoffs were calculated for all events in the UH dataset using the IGRF13 and Tsyganenko 05 internal/external geomagnetic field models. The resulting UH spectrum, Figure 8, incorporating all the corrections discussed above, and using the effective cutoff rigidity cut, shows a clear improvement in charge resolution in the $\mathrm{UH}$ region.

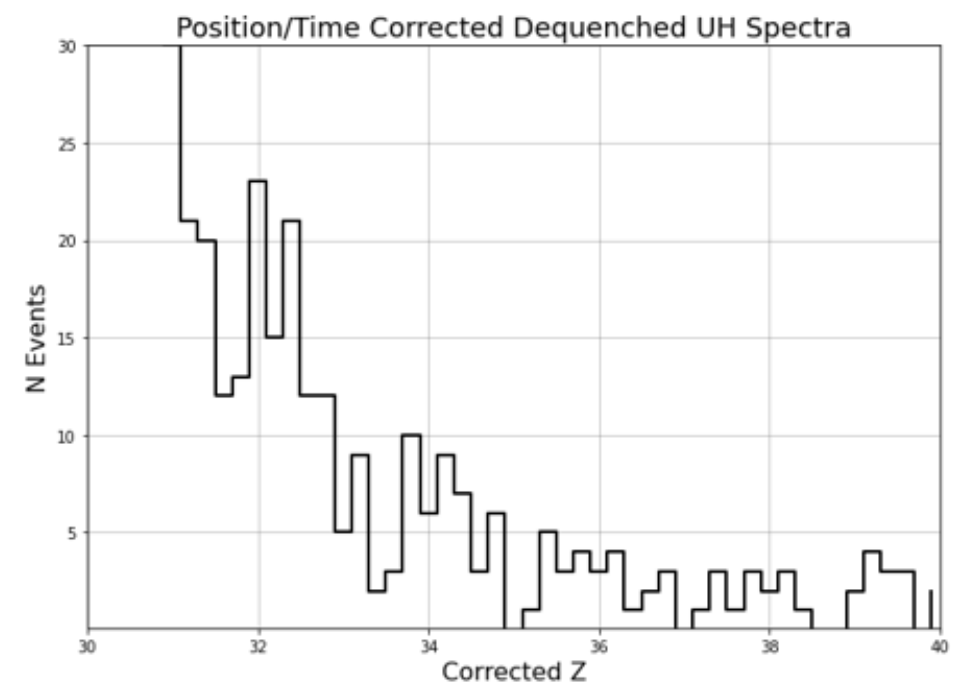

Figure 8: UH charge spectrum with effective cutoff rigidities $>4 \mathrm{GV}$. 


\section{Conclusions}

The analysis improvements described in this work have provided results consistent with previous CALET UH results, producing a UH spectrum with resolved peaks for evenly charged nuclei up to $\mathrm{Z}=38$. Using numerically computed effective cutoff rigidities to limit contamination in the UH dataset has improved charge resolution in the UH region. Further refinements to the analysis, including analysis presented by Zober et al. [6], along with increased statistics from the extended CALET mission will continue to improve CALET UH results going forward.

\section{Acknowledgements}

The material contained in this document is based upon work supported by a National Aeronautics and Space Administration (NASA) grant or cooperative agreement. Any opinions, findings, conclusions, or recommendations expressed in this material are those of the author and do not necessarily reflect the views of NASA. This work is supported in Japan by JAXA, in Italy by ASI and in the U.S. by NASA.

\section{References}

[1] Y. Asaoka, S. Ozawa, and S. Torii et al. (CALET Collaboration), On-orbit operations and offline data processing of CALET onboard the ISS, Astropart. Phys. 100, 29 (2018).

[2] P. S. Marrocchesi et al. (CALET Collaboration), New results from the first 5 years of CALET observations on the International Space Station in Proceedings of Science (ICRC2021; this conference) 786, (2021).

[3] B . F. Rauch and W. R. Binns et al. (CALET Collaboration), CALET Ultra Heavy Cosmic Ray Observations on the ISS, in Proceedings of Science of The 36th International Cosmic Ray Conference) 130, (2019).

[4] D. J. Hoffmann and H. H. Sauer, Magnetospheric Cosmic-Ray Cutoffs and their Variations, in Space Science Reviews, 8:750, (1968).

[5] D.F. Smart, Changes in calculated vertical cutoff rigidities at the altitude of the international space station as a function of magnetic activity, in Proceedings of Science ICRC 1990 7:337, (1990).

[6] W. Zober, B. F. Rauch, A. Ficklin and N. Cannady et al. (CALET Collaboration), Progress on Ultra-Heavy Cosmic-Ray Analysis with CALET on the International Space Station, in Proceedings of Science (ICRC2021; this conference) 1044, (2021). 


\section{Full Authors List: CALET Collaboration Collaboration}

O. Adriani ${ }^{1,2}$, Y. Akaike ${ }^{3,4}$, K. Asano ${ }^{5}$, Y. Asaoka ${ }^{5}$, E. Berti ${ }^{1,2}$, G. Bigongiari 6,7 , W. R. Binns ${ }^{8}$, M. Bongi ${ }^{1,2}$, P. Brogi ${ }^{6,7}$, A. Bruno ${ }^{9,10}$, J. H. Buckley ${ }^{8}$, N. Cannady ${ }^{11,12,13}$, G. Castellini ${ }^{14}$, C. Checchia ${ }^{6}$, M. L. Cherry ${ }^{15}$, G. Collazuol ${ }^{16,17}$, K. Ebisawa ${ }^{18}$, A. W. Ficklin ${ }^{15}$, H. Fuke ${ }^{18}$, S. Gonzi ${ }^{12}{ }^{1}$, T. G. Guzik ${ }^{15}$, T. Hams ${ }^{11}$, K. Hibino ${ }^{19}$, M. Ichimura ${ }^{20}$, K. Ioka $^{21}$, W. Ishizaki ${ }^{5}$, M. H. Israel ${ }^{8}$, K. Kasahara ${ }^{22}$, J. Kataoka ${ }^{23}$, R. Kataoka ${ }^{24}$, Y. Katayose ${ }^{25}$, C. Kato $^{26}$, N. Kawanaka ${ }^{27,28}$, Y. Kawakubo ${ }^{15}$, K. Kobayashi ${ }^{3,4}$, K. Kohri ${ }^{29}$, H. S. Krawczynski ${ }^{8}$, J. F. Krizmanic ${ }^{11,12,13}$,

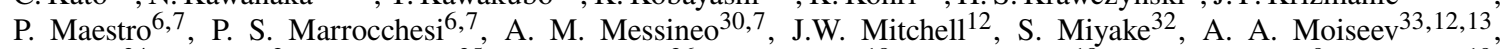
M. Mori ${ }^{34}$, N. Mori $^{2}$, H. M. Motz ${ }^{35}$, K. Munakata ${ }^{26}$, S. Nakahira ${ }^{18}$, J. Nishimura ${ }^{18}$, G. A. de Nolfo ${ }^{9}$, S. Okuno ${ }^{19}$, J. F. Ormes $^{36}$, N. Ospina ${ }^{16,17}$, S. Ozawa ${ }^{37}$, L. Pacini ${ }^{1,14,2}$, P. Papini ${ }^{2}$, B. F. Rauch ${ }^{8}$, S. B. Ricciarini ${ }^{14,2}$, K. Sakai ${ }^{11,12,13 \text {, }}$ T. Sakamoto ${ }^{38}$, M. Sasaki ${ }^{33,12,13}$, Y. Shimizu ${ }^{19}$, A. Shiomi ${ }^{39}$, P. Spillantini ${ }^{1}$, F. Stolzi ${ }^{6,7}$, S. Sugita ${ }^{38}$, A. Sulaj 6,7 , M. Takita ${ }^{5}$, T. Tamura ${ }^{19}$, T. Terasawa ${ }^{40}$, S. Torii ${ }^{3}$, Y. Tsunesada ${ }^{41}$, Y. Uchihori ${ }^{42}$, E. Vannuccini ${ }^{2}$, J. P. Wefel ${ }^{15}$, K. Yamaoka ${ }^{43}$, S. Yanagita ${ }^{44}$, A. Yoshida ${ }^{38}$, K. Yoshida $^{22}$, and W. V. Zober ${ }^{8}$

${ }^{1}$ Department of Physics, University of Florence, Via Sansone, 1, 50019 Sesto, Fiorentino, Italy, ${ }^{2}$ INFN Sezione di Florence, Via Sansone, 1, 50019 Sesto, Fiorentino, Italy, ${ }^{3}$ Waseda Research Institute for Science and Engineering, Waseda University, 17 Kikuicho, Shinjuku, Tokyo 162-0044, Japan, ${ }^{4}$ JEM Utilization Center, Human Spaceflight Technology Directorate, Japan Aerospace Exploration Agency, 2-1-1 Sengen, Tsukuba, Ibaraki 305-8505, Japan, ${ }^{5}$ Institute for Cosmic Ray Research, The University of Tokyo, 5-1-5 Kashiwa-no-Ha, Kashiwa, Chiba 277-8582, Japan, ${ }^{6}$ Department of Physical Sciences, Earth and Environment, University of Siena, via Roma 56, 53100 Siena, Italy, ${ }^{7}$ INFN Sezione di Pisa, Polo Fibonacci, Largo B. Pontecorvo, 3, 56127 Pisa, Italy, ${ }^{8}$ Department of Physics and McDonnell Center for the Space Sciences, Washington University, One Brookings Drive, St. Louis, Missouri 63130-4899, USA, ${ }^{9}$ Heliospheric Physics Laboratory, NASA/GSFC, Greenbelt, Maryland 20771, USA, ${ }^{10}$ Department of Physics, Catholic University of America, Washington, DC 20064, USA, ${ }^{11}$ Center for Space Sciences and Technology, University of Maryland, Baltimore County, 1000 Hilltop Circle, Baltimore, Maryland 21250, USA, ${ }^{12}$ Astroparticle Physics Laboratory, NASA/GSFC, Greenbelt, Maryland 20771, USA, ${ }^{13}$ Center for Research and Exploration in Space Sciences and Technology, NASA/GSFC, Greenbelt, Maryland 20771, USA, ${ }^{14}$ Institute of Applied Physics (IFAC), National Research Council (CNR), Via Madonna del Piano, 10, 50019 Sesto, Fiorentino, Italy, ${ }^{15}$ Department of Physics and Astronomy, Louisiana State University, 202 Nicholson Hall, Baton Rouge, Louisiana 70803, USA, ${ }^{16}$ Department of Physics and Astronomy, University of Padova, Via Marzolo, 8, 35131 Padova, Italy, ${ }^{17}$ INFN Sezione di Padova, Via Marzolo, 8, 35131 Padova, Italy, ${ }^{18}$ Institute of Space and Astronautical Science, Japan Aerospace Exploration Agency, 3-1-1 Yoshinodai, Chuo, Sagamihara, Kanagawa 252-5210, Japan, ${ }^{19}$ Kanagawa University, 3-27-1 Rokkakubashi, Kanagawa, Yokohama, Kanagawa 221-8686, Japan, ${ }^{20}$ Faculty of Science and Technology, Graduate School of Science and Technology,, Hirosaki University, 3, Bunkyo, Hirosaki, Aomori 036-8561, Japan, ${ }^{21}$ Yukawa Institute for Theoretical Physics, Kyoto University, Kitashirakawa Oiwakecho, Sakyo, Kyoto 606-8502, Japan, ${ }^{22}$ Department of Electronic Information Systems, Shibaura Institute of Technology, 307 Fukasaku, Minuma, Saitama 337-8570, Japan, ${ }^{23}$ School of Advanced Science and Engineering, Waseda University, 3-4-1 Okubo, Shinjuku, Tokyo 169-8555, Japan, ${ }^{24}$ National Institute of Polar Research, 10-3, Midori-cho, Tachikawa, Tokyo 190-8518, Japan, ${ }^{25}$ Faculty of Engineering, Division of Intelligent Systems Engineering, Yokohama National University, 79-5 Tokiwadai, Hodogaya, Yokohama 240-8501, Japan, ${ }^{26}$ Faculty of Science, Shinshu University, 3-1-1 Asahi, Matsumoto, Nagano 390-8621, Japan, ${ }^{27}$ Hakubi Center, Kyoto University, Yoshida Honmachi, Sakyo-ku, Kyoto 606-8501, Japan, ${ }^{28}$ Department of Astronomy, Graduate School of Science, Kyoto University, Kitashirakawa Oiwake-cho, Sakyo-ku, Kyoto 6068502, Japan, ${ }^{29}$ Institute of Particle and Nuclear Studies, High Energy Accelerator Research Organization, 1-1 Oho, Tsukuba, Ibaraki 305-0801, Japan, ${ }^{30}$ University of Pisa, Polo Fibonacci, Largo B. Pontecorvo, 3, 56127 Pisa, Italy, ${ }^{31}$ Astroparticle Physics Laboratory, NASA/GSFC, Greenbelt, Maryland 20771, USA, ${ }^{32}$ Department of Electrical and Electronic Systems Engineering, National Institute of Technology, Ibaraki College, 866 Nakane, Hitachinaka, Ibaraki 312-8508, Japan ${ }^{33}$ Department of Astronomy, University of Maryland, College Park, Maryland 20742, USA, ${ }^{34}$ Department of Physical Sciences, College of Science and Engineering, Ritsumeikan University, Shiga 525-8577, Japan, ${ }^{35}$ Faculty of Science and Engineering, Global Center for Science and Engineering, Waseda University, 3-4-1 Okubo, Shinjuku, Tokyo 169-8555, Japan, ${ }^{36}$ Department of Physics and Astronomy, University of Denver, Physics Building, Room 211, 2112 East Wesley Avenue, Denver, Colorado 80208-6900, USA, ${ }^{37}$ Quantum ICT Advanced Development Center, National Institute of Information and Communications Technology, 4-2-1 Nukui-Kitamachi, Koganei, Tokyo 184-8795, Japan, ${ }^{38}$ College of Science and Engineering, Department of Physics and Mathematics, Aoyama Gakuin University, 5-10-1 Fuchinobe, Chuo, Sagamihara, Kanagawa 252-5258, Japan, ${ }^{39}$ College of Industrial Technology, Nihon University, 1-2-1 Izumi, Narashino, Chiba 275-8575, Japan ${ }^{40}$ RIKEN, 2-1 Hirosawa, Wako, Saitama 351-0198, Japan, ${ }^{41}$ Division of Mathematics and Physics, Graduate School of Science, Osaka City University, 3-3-138 Sugimoto, Sumiyoshi, Osaka 558-8585, Japan, ${ }^{42}$ National Institutes for Quantum and Radiation Science and Technology, 4-9-1 Anagawa, Inage, Chiba 263-8555, Japan, ${ }^{43}$ Nagoya University, Furo, Chikusa, Nagoya 464-8601, Japan, ${ }^{44}$ College of Science, Ibaraki University, 2-1-1 Bunkyo, Mito, Ibaraki 310-8512, Japan 